\title{
APORTES PARA UNA EPIDEMIOLOGÍA DEL SUICIDIO EN COSTA RICA: UN ANÁLISIS DE LA PRIMERA DÉCADA DEL SIGLO XXI
}

\section{CONTRIBUTIONS TO A SUICIDAL EPIMEDIOLOGY IN COSTA RICA: XXI'S FIRST DECADE ANALYSIS}

\author{
Mario A. Sáenz Rojas*
}

RESUMEN

En este artículo se presentan diferentes datos estadísticos respecto a la epidemiología del suicidio en Costa Rica, durante el periodo 2000-2009. Los datos se dividieron en cuatro apartados para su tratamiento y análisis, estableciéndose, en la medida de lo posible, comparaciones con lo sucedido en otros países: magnitud del fenómeno, ocurrencia del fenómeno, género y localización geográfica. Para finalizar, se discuten algunos aspectos relacionados con la prevención y la necesidad de una política de salud mental, líneas de investigación para profundizar en esta área y la urgencia de trabajar en el eje de masculinidad $y$ violencia.

PALABRAS CLAVE: COSTA RICA * SUICIDIO * EPIDEMIOLOGÍA * SALUD MENTAL * CONDICIONES SOCIALES

\section{ABSTRACT}

This article presents statistical data regarding the suicidal epidemiology in Costa Rica, during the years 2000-2009. The information is divided into four categories for its thorough understanding and analysis, establishing as far as possible, comparisons with what has happened in other countries: dimension of the phenomenon, frequency of the phenomenon, gender and location. Finally, it presents a discussion on prevention and the need of a mental health policy, investigation to deepen the knowledge on this area and the urgency of work in the axis of masculinity and violence.

KEYWORDS: COSTA RICA * SUICIDE * EPIDEMIOLOGY * MENTAL HEALTH * SOCIAL CONDITIONS

Escuela de Psicología, Universidad Católica de

Costa Rica.

masaenz65@gmail.com 


\section{INTRODUCCIÓN}

El suicidio ha estado presente en la historia humana desde tiempos inmemoriales $y$ se ha asociado con ritos religiosos $y$ diversas manifestaciones visibles en la historia del arte $y$ de la política. Al respecto, de acuerdo con Taborda y Téllez-Vargas (s. f.), las tasas de suicidio varían en cada país, región y década, en relación directa con los múltiples factores que se asocian con la conducta suicida.

Es importante señalar que varios autores aluden a que la Organización Mundial de la Salud estimó un millón de muertes por suicidio para el año 2000 (Arán, Gispert, Puig, Freitas, Ribas y Puigdefâbregas, 2006; Gutiérrez-García, Contreras y Orozco-Rodríguez, 2006; Taborda y Téllez-Vargas, s. f.), lo cual ubica al suicidio como una frecuente causa de muerte.

Esta misma entidad ha descrito diversos factores de riesgo para la conducta suicida, entre los cuales se pueden mencionar el abuso del alcohol $y$ otras drogas, los antecedentes de abusos físicos o sexuales en la infancia, el aislamiento social, los problemas psiquiátricos (particularmente depresión, otros trastornos del estado de ánimo y la esquizofrenia), un sentimiento generalizado de desesperanza, las enfermedades de base somática (sobre todo las dolorosas o discapacitantes), el acceso a los medios para auto-eliminarse; estos factores potencian algunos estresores ambientales, tales como la pobreza, el desempleo, la pérdida de personas queridas, las discusiones con la familia o los amigos, las ruptura de las relaciones y los problemas legales o laborales (Krug, Drahiberg, Mercy, Zwi y Lozano, 2003).

Cabe iniciar este apartado, citando la clásica definición de Durkheim, citado por Lastra, Rodríguez, González y Vázquez-Barquero (1999), quien considera que suicidio es "... todo caso de muerte que resulta directa o indirectamente de un acto positivo o negativo, ejecutado por la propia víctima a sabiendas de que tal era el resultado que se produciría" (p. 753).

De esta manera, surge la necesidad de cuestionarse sobre el perfil epidemiológico del suicidio en Costa Rica, en tanto la epidemiología se encarga de estudiar la distribución y frecuencia de la patología en el ser humano, los factores que la condicionan y evalúa los programas de prevención primaria, secundaria y terciaria (De Galvis, 1990). De esta forma, el presente artículo intenta adentrarse en los dos primeros niveles de esta definición; o sea, en el diagnóstico y el conocimiento de los factores de riesgo.

Dicho cuestionamiento perseguiría tres fines, a saber: disminuir los casos nuevos de actos parasuicidas, disminuir la cantidad de suicidios consumados y aumentar la esperanza de vida y su calidad.

En este contexto, es menester ubicar la realidad costarricense para el año 2009, en el cual contaba con una población de 4509 392 habitantes, una tasa bruta de mortalidad de 3,4 y una densidad poblacional de 140,71 habitantes por $\mathrm{km}^{2}$ (Proyecto Estado de la Nación, 2010).

Seguidamente se expondrán los hallazgos de algunas investigaciones sobre el tema, tanto en el plano nacional como internacional.

\section{ANTECEDENTES}

En el caso español, Lastra et ál. (1999) indican que entre 1976 y 1991 la tasa de suicidios por cien mil habitantes osciló entre 3,6 y 5,3 con un leve descenso entre 1980-1982 pero conservando una marcada tendencia al alza.

Asimismo, diversos estudios (Montaro, 1997; Legra y Aces, 1998; citados por Matos, Betancourt, Álvarez, Aces y Toirac, 2001) refieren a estadísticas internacionales en las que se destaca que un tercio de las muertes por suicidio está relacionado con el consumo de alcohol.

En este sentido, Matos et ál. (2001) registraron las alcoholemias positivas encontradas en todos los casos de muerte violenta ocurridos en la provincia de Guantánamo, Cuba, durante el periodo 19951998 y determinaron la presencia de alcohol en una cuarta parte de los suicidios analizados.

En el caso costarricense, Bejarano y Sáenz (2000) efectuaron un estudio en el año 1996, en el cual determinaron, según el criterio del médico forense, una clara asociación entre la muerte por suicidio y el consumo de bebidas 
alcohólicas, registrándose concentraciones de alcohol en sangre de $120 \mathrm{mg}$. o superiores en dos terceras partes de los suicidas.

Lo anterior concuerda con varios estudios citados por Díaz y Sanabria (1994), en los que se afirma que entre drogadictos, la incidencia de tentativas de suicidio o actos parasuicidas y de pensamientos suicidas es significativamente mayor que en la población normal.

Particularizando en la dimensión epidemiológica del suicidio en Costa Rica, Granados (1997) determinó varios aspectos de interés a nivel nacional para el periodo comprendido entre 1980 y 1994:

$\diamond \quad$ Se registró una tasa promedio anual de suicidios de 4,8 por cada cien mil habitantes.

$\diamond \quad$ Solo un $10 \%$ de los fallecidos por suicidio en el periodo comprendido entre 1980 y 1994 se trataba de personas menores de 20 años de edad.

$\diamond \quad$ La edad promedio de las personas que se suicidaron fue de 35 años en el trienio 1980-1982 y de 38 años en el trienio 1992-1994, siendo que cada una de estas personas dejó de aportar 41 y 38 años de vida en cada trienio.

$\diamond \quad$ Este mismo estudio señaló que el 84,2\% de los suicidas en dicho periodo fueron varones, contra un $15,8 \%$ de mujeres.

$\diamond \quad$ Los intervalos de edad que registraron una mayor tasa anual promedio de suicidios por cada cien mil habitantes, fueron el de 30-39 años y el de 75 años $y$ más.

$\diamond \quad$ A pesar de esto, la tasa de suicidios por cada cien mil habitantes tiende a incrementarse levemente en un trienio $y$ a disminuir en el trienio siguiente.

Siempre aludiendo al periodo 1980-1994, al desagregar los datos por provincia (Granados, 1998a) y por cantón (Granados, 1998b) halló los siguientes aspectos relevantes:

$\diamond \quad$ Las tres provincias que presentaron mayor proporción de suicidios fueron en su orden: San José (39\%), Alajuela (17\%) y Limón (11\%).

$\diamond \quad$ La tasa promedio anual más alta de mortalidad por suicidio corresponde a la provincia de Limón con 7,3 suicidios por cada cien mil habitantes, seguida por San José $(5,1)$ y Alajuela $(4,9)$, cifra bastante superior al promedio nacional $(4,8)$.

$\diamond \quad$ A pesar de que la provincia de Limón registra una tasa promedio anual de suicidios para el primer trienio (19801982) de 5,5 y de 7,2 para el quinto trienio (1992-1994), llegó a presentar en los trienios intermedios hasta un 8,4 por cada cien mil habitantes; mientras tanto, Guanacaste presentó un ascenso importante en su tasa promedio por trienio de 3,3 en el primero a 5,4 en el último trienio.

$\diamond \quad$ De los 19 cantones que integran la provincia de San José, un total de 12 presentan una tasa promedio anual de suicidios por encima del promedio nacional $(4,8)$, siendo los tres primeros: Dota $(13,4)$, Tarrazú $(12,1)$ y León Cortés $(10,9)$.

$\diamond \quad$ De los 15 cantones que integran la provincia de Alajuela, ocho registran una tasa promedio anual de suicidios superior al promedio nacional, siendo los primeros tres: Poás $(7,0)$, Naranjo $(6,3)$ y Cantón Central de Alajuela $(6,0)$.

$\diamond \quad$ De los ocho cantones que componen la provincia de Cartago, solo tres tienen una tasa promedio anual de suicidios superior al promedio nacional, siendo estos cantones: Alvarado (10,1), Paraíso $(5,1)$ y Turrialba $(4,9)$.

$\diamond \quad$ De los 10 cantones que integran la provincia de Heredia, tres de ellos registran una tasa promedio anual de suicidios por encima del promedio nacional, siendo estos cantones: San Isidro $(7,1)$, Santa Bárbara $(5,5)$ y Santo Domingo $(5,2)$.

$\diamond \quad$ De los 11 cantones que componen la provincia de Guanacaste, seis de ellos presentan una tasa promedio anual 
de suicidios que supera el promedio nacional, siendo los tres primeros cantones: Abangares (7,9), Cañas $(7,0) y$ Bagaces $(6,2)$.

$\diamond \quad$ De los 11 cantones que integran la provincia de Puntarenas, cuatro tienen una tasa promedio anual de suicidios mayor al promedio nacional, siendo los primeros tres: Garabito $(5,7)$, Coto Brus $(5,6)$ y Buenos Aires $(5,3)$.

$\diamond \quad$ Por último, sobresale que los seis cantones que componen la provincia de Limón superaron la tasa anual promedio de suicidios a nivel nacional, siendo los tres que más destacaron: Pococí $(9,5)$, Talamanca $(8,1)$ y Matina $(7,9)$.

$\diamond \quad$ De esta manera, los 10 cantones con mayor tasa promedio anual de suicidios son los siguientes: Dota (13,4), Tarrazú $(12,1)$, León Cortés $(10,9)$, Alvarado $(10,1)$, Pococí $(9,5)$, Talamanca $(8,1)$, Matina (7,9), Abangares (7,9), Guácimo $(7,3)$ y Cañas $(7,0)$.

Por otra parte, Chanto $(2004 / 2005)$ investigó a los y las adolescentes que ingresaron al Hospital Nacional Psiquiátrico en julio de 2004 por realizar alguna conducta suicida; al respecto determinó que la mayoría de adolescentes que efectuaron intentos de autoeliminación fueron mujeres, sin embargo, predominaron los gestos suicidas, debido a que la mayoría de las acciones desplegadas no tuvieron secuelas físicas de importancia.

La autora también identificó:

... que las conductas suicidas en la población adolescente; si no son tratadas de una manera adecuada y oportuna, se pueden convertir en una conducta crónica y repetitiva que con el paso del tiempo puede llegar a aumentar en el grado de peligro y auto destrucción (Chanto, 2004/2005: 29).

Asimismo, Chanto (2004/2005) estableció que estas conductas parasuicidas se encuentran sustentadas por un predominio de las creencias irracionales en torno a la muerte, atravesadas por pensamientos negativos e ilógicos.

\section{RECOPILACIÓN DE LOS DATOS}

Para la confección del presente artículo los datos se recopilaron de los Anuarios de Estadísticas Policiales del Poder Judicial de manera digital $y$ en algunos casos, se efectuaron los cálculos respectivos para el establecimiento de frecuencias relativas.

\section{MAGNITUD DEL PROBLEMA}

La tendencia del suicidio en números absolutos a lo largo de la década (2000-2009) evidencia que a partir del año 2003 los suicidios superaron los 300 anuales $y$ a partir de dicho año, con excepción de 2006 y 2009, la cifra guarda una relativa estabilidad (véase la figura 1 ).

\section{FIGURA 1}

TENDENCIA DEL NÚMERO DE FALLECIMIENTOS POR SUICIDIO COSTA RICA, 2000-2009 (VALORES ABSOLUTOS)

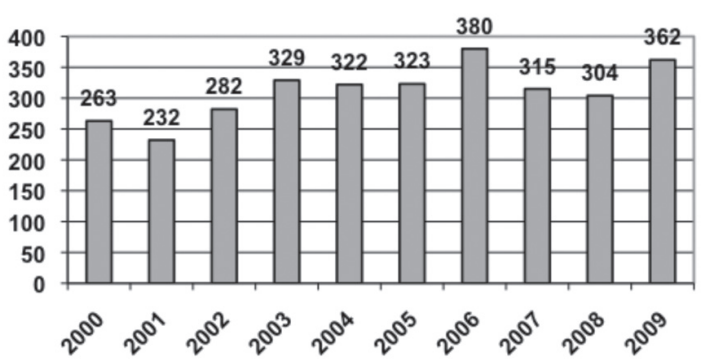

Fuente: Anuarios de Estadísticas Policiales, Poder Judicial. En: <http://www.poder-judicial.go.cr/planificacion/ Estadisticas/policiales.html>

En la tabla 1 se presenta la tasa de suicidios por cien mil habitantes, correspondiente a la población total de Costa Rica y su respectiva segregación por sexo, (véase tabla 1).

A lo largo de la década, se aprecia un ascenso leve pero constante, equivalente a 1,5 por cada cien mil habitantes entre ambos extremos del periodo; aunado a ello, para 1980 la tasa general fue de 4,2 por cada cien mil habitantes. Asimismo, resulta evidente la diferencia entre ambos sexos, la cual asciende durante la década a un aproximado de 10 por cada cien mil habitantes entre varones $y$ mujeres, aspecto que para la década de 1990 
TABLA 1

TASA POR 100000 HABITANTES DE LA POBLACIÓN FALLECIDA POR SUICIDIO, POR AÑO DE OCURRENCIA, SEGÚN SEXO

\begin{tabular}{lccc}
\hline AÑO & GENERAL & MASCULINO & FEMENINO \\
\hline 2000 & 6,7 & 11,6 & 1,6 \\
2001 & 5,8 & 10,1 & 1,4 \\
2002 & 7 & 12,0 & 1,8 \\
2003 & 7,9 & 13,3 & 2,2 \\
2004 & 7,7 & 13,1 & 2,2 \\
2005 & 7,7 & 12,9 & 2,2 \\
2006 & 8,9 & 14,8 & 2,9 \\
2007 & 7,3 & 11,9 & 2,5 \\
2008 & 6,9 & 11,9 & 1,9 \\
2009 & 8,2 & 13,8 & 2,4 \\
\hline PROMEDIO & 7,4 & 12,5 & 2,1 \\
\hline
\end{tabular}

Fuente: Anuarios de Estadísticas Policiales, Poder Judicial. En: <http://www.poder-judicial.go.cr/planificacion/ Estadisticas/policiales.html>

fue compartido por otros países de la región latinoamericana, tal es el caso de: Argentina, Chile, Cuba, El Salvador, Panamá, Puerto Rico, Uruguay y Venezuela (véase Tabla 2).
Siempre en el plano internacional, dicha tabla muestra la tasa de suicidios ajustada por edad en varios países de la región latinoamericana durante la década de 1990, denotándose que Costa Rica ocupa un lugar intermedio comparativamente hablando; de hecho, Costa Rica no aparece entre los países en que se suicidan más hombres por cada mujer, aunque aparece cerca del valor 5 , destacándose en orden descendente: Puerto Rico, Chile, México, Panamá y Venezuela.

Desde una perspectiva más reciente, Taborda y Tellez-Vargas (s. f.), refieren que la tasa anual de suicidios en Estados Unidos, para el año 2003, se estableció en 10,8 por cada cien mil habitantes. Más aún, los mismos autores plantean para el caso colombiano en el año 2005 , una tasa de 3,9 suicidios por cada cien mil habitantes, reduciéndose en un punto para el periodo 2000-2005. Además, en el caso de Chile, Moyano y Barria (2006) repotaron la tasa para el año 2003 en 8,8.

Por otra parte, la tasa anual promedio se estableció en 7,4 por cada cien mil habitantes, la cual supera en 2,6 la establecida por Granados (1997) para el periodo 1980-1994. De

TABLA 2

TASAS DE SUICIDIO POR CADA 100000 HABITANTES EN AMÉRICA LATINA, AJUSTADAS POR EDAD, POR PAÍS, SEGÚN AÑO, SEXO Y RAZÓN

\begin{tabular}{lccccc}
\hline PAÍS & AÑO & TASA GLOBAL & TASA VARONES & TASA MUJERES & $\begin{array}{c}\text { RAZÓN ENTRE VARONES/ } \\
\text { MUJERES }\end{array}$ \\
\hline Argentina & 1996 & 8,7 & 14,2 & 3,9 & 3,6 \\
Brasil & 1995 & 6,3 & 10,5 & 2,5 & 4,1 \\
Chile & 1994 & 8,1 & 15 & 1,9 & 8,1 \\
Colombia & 1995 & 4,5 & 7,4 & 1,8 & 4,1 \\
Costa Rica & 1995 & 8,8 & 14,4 & 3 & 4,7 \\
Cuba & 1997 & 23 & 32,1 & 14,2 & 2,3 \\
Ecuador & 1996 & 7,2 & 10,4 & 4,1 & 2,4 \\
El Salvador & 1993 & 11,2 & 16,3 & 6,8 & 6,3 \\
México & 1997 & 5,1 & 9,1 & 1,4 & 2,6 \\
Nicaragua & 1996 & 7,6 & 11,2 & 4,3 & 5,7 \\
Panamá & 1997 & 7,8 & 13,2 & 2,3 & 3,6 \\
Paraguay & 1994 & 4,2 & 6,5 & 1,8 & 10,4 \\
Puerto Rico & 1998 & 10,8 & 20,9 & 2 & 4,8 \\
Uruguay & 1990 & 12,8 & 22 & 4,8 & 5 \\
Venezuela & 1994 & 8,1 & 13,7 & 2,7 & \\
\hline
\end{tabular}

Fuente: Krug, Drahiberg, Mercy, Zwi y Lozano, 2003: 202-203. 
acuerdo con los parámetros establecidos por la Organización Mundial de la Salud (Tuesca y Navarro, 2003), estas tasas nacionales, tanto a nivel general como por sexo, pueden ser consideradas medias al encontrarse entre 5 y 14 por cada cien mil habitantes.
En la figura 2 se establece la comparación entre la tasa por cada cien mil habitantes de víctimas de suicidio, víctimas de homicidio doloso y víctimas de homicidio culposo.

FIGURA 2

COMPARACIÓN DE LA TASA DE SUICIDIOS, HOMICIDIOS DOLOSOS Y CULPOSOS

POR 100000 HABITANTES

COSTA RICA, 2000-2009
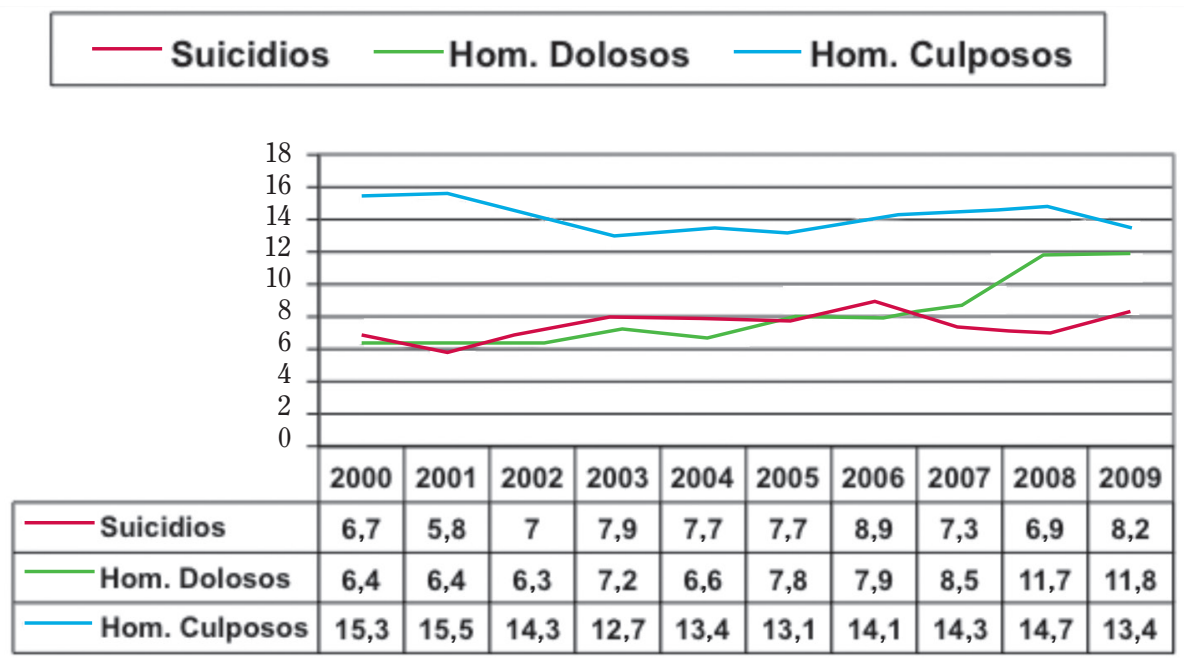

Fuente: Anuarios de Estadísticas Policiales, Poder Judicial.

En: <http://www.poder-judicial.go.cr/planificacion/Estadisticas/policiales.html>

Se aprecia que durante la primera década del segundo milenio, los homicidios culposos ocupan el primer lugar en muertes violentas $y$ a pesar de que se aprecia un descenso a partir de 2002, el mismo no es marcado y se mantiene relativamente estable. En cuanto a la relación entre suicidios $y$ homicidios dolosos, pareciera que hasta 2006 los primeros superaron levemente a los segundos, pero a partir de 2007 dicha relación se invirtió y tendió a separarse en niveles sensiblemente apreciables, colocando a los suicidios como la tercera causa de muerte violenta a nivel nacional.

En cuanto a la edad de las víctimas de suicidio a lo largo de la década, se puede observar la Tabla 3.

Se nota que los intervalos de 20 a 29 años y 30 a 39 años ocupan los principales porcentajes; en este sentido llama la atención que el periodo de la adolescencia no sobresalga entre los grupos de edad, pues existe una creencia de que el suicidio es una conducta típica de esta etapa del ciclo vital estereotipada como "problemática" desde visiones adulto-céntricas. Aunado a ello, es importante evaluar las condiciones sociales en que se desenvuelven las personas adultas-jóvenes en Costa Rica, caracterizadas por expectativas de alto estatus, escasas oportunidades laborales $y$ de ingresos satisfactorios, el acelerado consumismo, la existencia de ideales de autorealización sobrevalorados como metas socialmente aceptadas $y$ la inaccesibilidad a mecanismos válidos para alcanzar tales metas, entre otros aspectos.

No obstante, lo anterior debe rescatarse lo aportado por Chanto $(2004 / 2005)$ en 
TABLA 3

EDAD DE LA POBLACIÓN FALLECIDA POR SUICIDIO, POR GRUPO ETARIO, SEGÚN AÑO

COSTA RICA, 2000-2009

(VALORES PORCENTUALES)

\begin{tabular}{lcccccccccc}
\hline EDAD EN AÑOS & 2000 & 2001 & 2002 & 2003 & 2004 & 2005 & 2006 & 2007 & 2008 & 2009 \\
\hline Menos de 18 & 6,1 & 4,3 & 11,3 & 7,6 & 6,2 & 5,3 & 6,3 & 5,7 & 5,6 & 3,6 \\
De 18 a 19 años & 3,8 & 3,9 & 6,0 & 3,6 & 5,9 & 5,0 & 5,8 & 5,7 & 4,6 & 3,6 \\
De 20 a 29 años & 28,2 & 27,6 & 23,4 & 27,5 & 23,6 & 23,8 & 26,0 & 26,8 & 25,2 & 29,9 \\
De 30 a 39 años & 20,5 & 25,0 & 21,3 & 22,5 & 20,2 & 22,9 & 18,9 & 20,5 & 21,4 & 19,9 \\
De 40 a 49 años & 19,8 & 17,7 & 16,7 & 16,7 & 20,2 & 21,0 & 22,1 & 21,1 & 18,8 & 18,5 \\
De 50 a 59 años & 9,5 & 9,0 & 11,0 & 11,8 & 12,7 & 12,1 & 12,1 & 11,7 & 14,8 & 13,5 \\
De 60 a 69 años & 5,3 & 6,0 & 3,9 & 6,1 & 5,9 & 6,5 & 6,2 & 6,3 & 5,3 & 6,6 \\
70 y más años & 6,8 & 6,5 & 6,4 & 4,2 & 5,3 & 3,4 & 2,6 & 2,2 & 4,3 & 4,4 \\
\hline TOTAL & $100 \%$ & $100 \%$ & $100 \%$ & $100 \%$ & $100 \%$ & $100 \%$ & $100 \%$ & $100 \%$ & $100 \%$ & $100 \%$ \\
\hline
\end{tabular}

Fuente: Anuarios de Estadísticas Policiales, Poder Judicial. En: <http://www.poder-judicial.go.cr/planificacion/Estadisticas/ policiales.html>

el sentido de que las conductas suicidas en personas adolescentes pueden asumir con el paso del tiempo un mayor nivel de letalidad si no se efectúa una intervención adecuada $y$ oportuna.

FIGURA 3

DISTRIBUCIÓN PROMEDIO POR GRUPO DE EDAD DE LA POBLACIÓN FALLECIDA POR SUICIDIO COSTA RICA, 2000-2009 (VALORES PORCENTUALES)

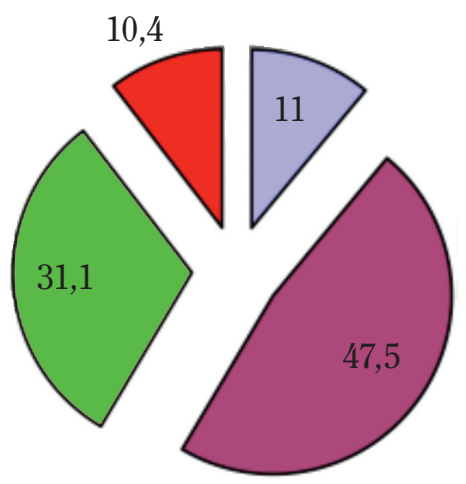

$$
\begin{array}{ll}
\hline \text { Menos de } 20 \text { años } & \square 20 \text { a } 39 \text { años } \\
\square \text { 40 a } 59 \text { años } & \square \quad 60 \text { y más años }
\end{array}
$$

Fuente: Elaboración personal.

Al analizar el conjunto del periodo, se destaca que cerca de la mitad de las personas fallecidas por suicidio se ubican entre los 20 y los 39 años, periodo de la vida en el que se supone que las personas atraviesan por un estadio de estabilidad y consolidación de su proyecto de vida $y$ alrededor de un tercio entre los 40 y los 59 años; mientras tanto, en Colombia, para el año 2006, más de la mitad de quienes fallecieron por suicidio se ubicaban entre los 18 y los 44 años de edad (Taborda y Téllez-Vargas, 2006). Además, los periodos de infancia-adolescencia (menos de 20 años) y de adultez mayor (60 y más años) obtuvieron cifras bastante similares, alcanzando en conjunto cerca de una quinta parte de la población suicida; lo anterior es un elemento que de alguna manera no apoya la posición alarmista de Vargas (1997) en torno al suicidio en personas menores de edad.

Llama la atención que en el caso del estudio de Granados (1997) la edad promedio de las personas fallecidas por suicidio se estableció en 35 años para el primer trienio del periodo 1980-1994, mientras que para el último trienio se estimó en 38 años, pues dos tercios (66,8\%) de la población fallecida por suicidio entre 2000 y 2009 se ubican entre los 20 y los 49 años. Aunado a ello, en ese mismo estudio (Granados) la población menor de 20 años de edad alcanzó un $10 \%$, cifra similar a la presentada en la figura 3 .

En este sentido, es de suma importancia retomar la pérdida de años de vida y su impacto negativo en la proporción de la población 
económicamente activa (PEA) y por ende, en el producto interno bruto (PIB). Al respecto, Méndez y Araya (2001) establecieron para el año 2000 una esperanza de vida al nacer de 74,81 años para los varones $y$ de 80,29 años para las mujeres. Lo anterior sugiere, al menos, una pérdida promedio de 38 y 44 años de vida para los varones y las mujeres respectivamente. Al respecto, es importante señalar que Weinstein y Saturno (1989) citados en Lastra et ál. (1999), establecieron que cada suicidio de un joven de 15 a 24 años de edad en Estados Unidos supone una pérdida de 53 años de vida $y$ alrededor de \$ 432000 de productividad; igualmente, Taborda y Téllez-Vargas (2006), refieren que en Colombia, para el año 2005 se perdieron más de 55000 años potenciales de vida.

A lo largo de los diez años es constante que más del $85 \%$ de las personas fallecidas por suicidio fuesen costarricenses (véase la tabla 4).

TABLA 4

NACIONALIDAD DE LA POBLACIÓN FALLECIDA POR SUICIDIO, POR CONDICIÓN DE PAÍS DE NACIMIENTO, SEGÚN AÑO COSTA RICA, 2000-2010 (VALORES PORCENTUALES)

\begin{tabular}{lcccccccccc}
\hline NACIONALIDAD & 2000 & 2001 & 2002 & 2003 & 2004 & 2005 & 2006 & 2007 & 2008 & 2009 \\
\hline Costarricense & 89,7 & 85,8 & 87,6 & 89,7 & 87,6 & 88,2 & 86,3 & 86,3 & 87,5 & 88,1 \\
Extranjero & 10,3 & 14,2 & 12,4 & 10,3 & 12,4 & 11,8 & 13,2 & 13,7 & 12,5 & 11,9 \\
Ignorado & --- & --- & --- & --- & --- & --- & 0,5 & --- & --- & --- \\
\hline TOTAL & $100 \%$ & $100 \%$ & $100 \%$ & $100 \%$ & $100 \%$ & $100 \%$ & $100 \%$ & $100 \%$ & $100 \%$ & $100 \%$ \\
\hline
\end{tabular}

Fuente: Anuarios de Estadísticas Policiales, Poder Judicial. En:< http://www.poder-judicial.go.cr/planificacion/ Estadisticas/policiales.html>

Mientras tanto, en la figura 4 se aprecia la distribución de personas nicaragüenses fallecidas por suicidio durante la década (ver fig. 4).

A excepción del año 2001, los restantes representan menos del $10 \%$ de la población general, salvo el caso del año 2006, más de la mitad de extranjeros fallecidos. Al respecto llama la atención que para 2005 , las personas de origen nicaragüense representaban un $74,8 \%$ del total de población extranjera residente en el país y un $5,7 \%$ de la población total de Costa Rica.

Lo anterior denota una especial asociación entre esta población y la conducta suicida, situación que necesariamente debe analizarse en función de los procesos migratorios, el abandono de sus familias en su país de origen, las precarias condiciones de existencia en que habitan en Costa Rica y tal como se ha señalado en relación con el chiste (Bonilla y Sáenz, 2010), la discriminación de la cual son objeto estas personas, entre otros elementos.

$\mathrm{Al}$ respecto señala Tamarit: ...una particularidad de la condición de migrante como fuente de riesgo de victimización (...) es que esta condición está intrínsecamente asociada a una condición objetiva de ciudadanía disminuida, a un estatuto jurídico especial que lo convierte en 'no persona', en expresión de Brandariz, a partir de la idea de que sólo la ciudadanía atribuye personalidad social (2010: 11-12).

Esto resulta en un aporte significativo en relación con la visión fatalista del suicida, la cual se expresa por medio de pensamientos asociados con el fracaso y la inadecuación, afectos que denotan una auto-percepción negativa $y$ desvalorizada, así como, comportamientos que lesionan su autoestima y tienen un carácter fundamentalmente auto-destructivo.

Por otra parte, se ha señalado que la migración, con una serie de problemas asociados, tales como la pobreza extrema, las condiciones habitacionales paupérrimas y de hacinamiento, la carencia de redes de apoyo 
FIGURA 4

DISTRIBUCIÓN DE LA POBLACIÓN NICARAGÜENSE, POR REPRESENTACIÓN EN EL TOTAL DE SUICIDIOS Y EN EL TOTAL DE EXTRANJEROS, SEGÚN AÑO COSTA RICA, 2000-2010

(VALORES PORCENTUALES)

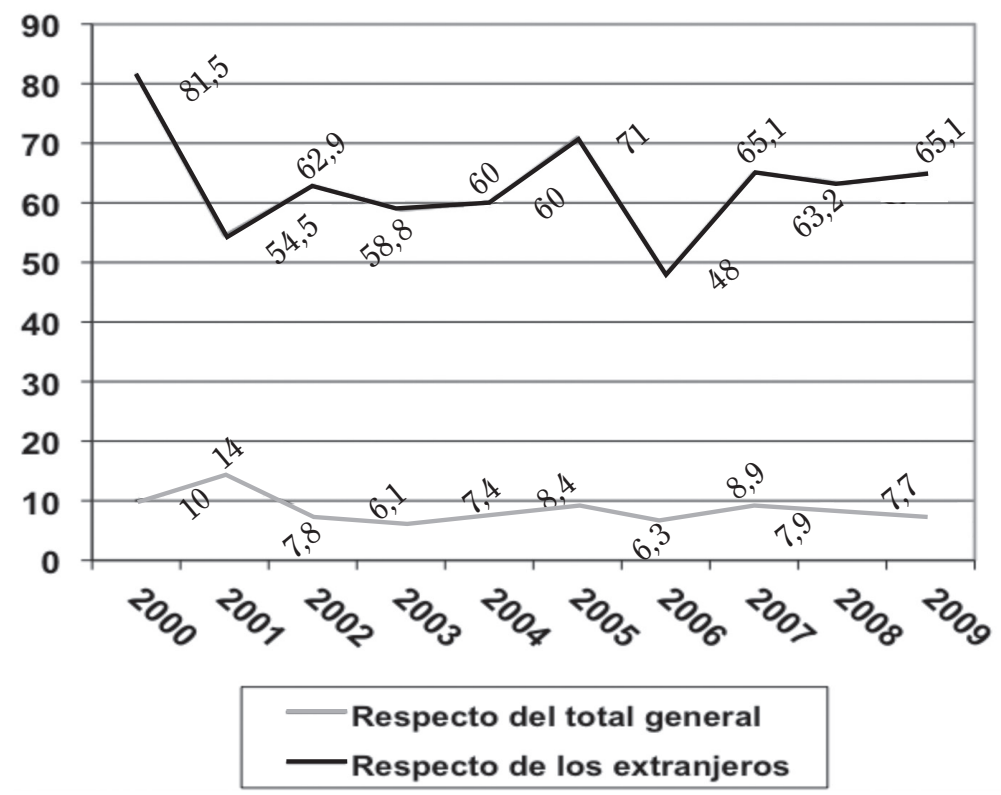

Fuente: Elaboración personal.

social y una serie de expectativas insatisfechas, incrementan el riesgo de suicidio (Taborda $y$ Téllez-Vargas, 2006).

En la tabla que aparece en la página siguiente se presentan los motivos policiales de los diferentes suicidios; resulta destacable que prácticamente todos los motivos tenían la particularidad de ser susceptibles de intervención desde una óptica psicosocial, no necesariamente para solucionar los problemas, pero sí para buscar alternativas viables de resolución 0 afrontamiento de las diferentes problemáticas.

De esta manera, resulta que en siete de los diez años contemplados, la categoría de problemas conyugales superó una cuarta parte de las personas fallecidas por suicidio. Asimismo, se destaca que los problemas asociados con el consumo de bebidas alcohólicas y otras drogas llevan un ritmo ascendente en el transcurso de la década.
Además, en cuanto a la categoría de problemas familiares, esta oscila entre 8,8 en 2003 y 14,3 en 2007, y la de problema mental se mantuvo relativamente estable, entre 6,1 y 7,7 con excepción de tres de los diez años. Otro aspecto interesante es que salvo cuatro de los diez años, en los restantes la categoría de problemas depresivos superó un 5\%.

En muchos de estos casos la ayuda psicológica oportuna y la de otros profesionales de la salud mental, podría haber tenido un carácter preventivo, lo cual remite a cuestionarse sobre la presencia de estos recursos profesionales en instituciones estatales del sector salud, social y educación, de manera que tales servicios lleguen a la mayoría de la población nacional.

Respecto a la depresión, también este factor ha sido considerado por Bertolote, Fleischmann, De Leo y Wasserman (2003), citados por Gutiérrez-García et ál. (2006), particularmente 
TABLA 5

DISTRIBUCIÓN DE LA POBLACIÓN FALLECIDA POR SUICIDIO, POR MOTIVO O CAUSA DEL EVENTO

SEGÚN AÑO COSTA RICA, 2000-2009

(VALORES PORCENTUALES)

\begin{tabular}{lrrrrrrrrrr}
\hline \multicolumn{1}{c}{ MOTIVO } & 2000 & 2001 & 2002 & 2003 & 2004 & 2005 & 2006 & 2007 & 2008 & 2009 \\
\hline Depresión por duelo o soledad & 5,3 & 3,9 & 5,7 & 8,5 & 5,3 & 2,1 & 2,1 & 6,4 & 4,6 & 8,0 \\
Problema pasional & 11,4 & 9,0 & 12,8 & 9,7 & 9,6 & 5,3 & 8,2 & 7,3 & 11,8 & 7,5 \\
Problema familiar & 9,1 & 12,9 & 12,0 & 8,8 & 9,0 & 12,7 & 11,6 & 14,3 & 11,5 & 10,5 \\
Problema mental & 6,1 & 9,5 & 11,7 & 6,7 & 6,5 & 7,7 & 14,5 & 7,6 & 6,3 & 6,4 \\
Problema con licor o droga & 9,1 & 8,6 & 8,1 & 13,4 & 9,9 & 16,1 & 15,3 & 11,4 & 14,5 & 14,1 \\
Problema económico & 12,2 & 5,6 & 6,0 & 6,4 & 8,7 & 5,9 & 2,9 & 5,4 & 7,2 & 5,5 \\
Problema de salud & 8,0 & 11,6 & 6,4 & 7,9 & 6,8 & 5,3 & 7,4 & 9,5 & 5,6 & 8,3 \\
Problema conyugal & 32,3 & 23,3 & 26,2 & 26,1 & 28,6 & 29,4 & 23,2 & 26,0 & 22,7 & 21,8 \\
Problema con la justicia & 2,3 & 2,6 & 2,1 & 4,2 & 4,0 & 2,8 & 6,3 & 6,3 & 3,6 & 4,7 \\
Problema personal & 3,4 & 7,8 & 3,2 & 4,2 & 4,0 & 7,7 & 3,9 & 3,5 & 7,6 & 2,5 \\
Otros problemas o situaciones & 0,8 & 0,4 & 2,2 & 1,2 & 0,9 & 0,6 & 1,8 & 2,2 & 0,7 & 1,2 \\
Ignorado & ---- & 4,7 & 4,2 & 2,7 & 6,5 & 4,3 & 2,9 & --- & 3,9 & 9,7 \\
\hline \multicolumn{1}{c}{ TOTAL } & $100 \%$ & $100 \%$ & $100 \%$ & $100 \%$ & $100 \%$ & $100 \%$ & $100 \%$ & $100 \%$ & $100 \%$ & $100 \%$ \\
\hline
\end{tabular}

Fuente: Anuarios de Estadísticas Policiales, Poder Judicial. En: <http://www.poder-judicial.go.cr/planificacion/Estadisticas/ policiales.html>

cuando se asocia con impulsividad y agresividad. En este sentido, Goldston, Reboussin y Sergent (2006) determinaron en la población adolescente una asociación entre altos grados de depresión, desesperanza, ansiedad e intentos de suicidio post-hospitalización.

En relación con la ruta ascendente registrada por la categoría de consumo de bebidas alcohólicas y otras drogas, es importante acotar que alrededor de una tercera parte de los varones $y$ un quinto de las mujeres que intentan suicidarse abusan de las bebidas etílicas (Koller, Preuss, Bottlender, Wenzel y Soyka, 2002, citados en Gutiérrez-García, 2006); en este mismo sentido se determinó que un tercio había consumido alcohol en las seis horas previas al evento (Borges, Cherpitel, Macdonald, Giesbrecht, Stockwell y Wilcox, 2004, citados por Gutiérrez-García et ál.).

Aunado a ello, de acuerdo con Inskip, Harris y Barraclough (1998); Suominen, Isometsa, Henriksson, Ostamo y Lonnqvist (1997), citados por Gutiérrez-García et ál. (2006) aproximadamente una décima parte de personas alcohólicas fallecen por suicidio. Este elemento se relaciona con la asociación estadísticamente significativa encontrada por Bejarano y Sáenz (2000), entre la muerte por suicidio y el consumo de alcohol, según el criterio del médico forense.

Merece citarse el estudio de Matos et ál. (2001), en el que refieren que en Guantánamo, Cuba, se presentó una cuarta parte de alcoholemias positivas en casos de suicidio, así como, del Instituto Nacional de Medicina Legal de Colombia, el cual reportó en 1998, alcoholemias positivas en varios departamentos del país, mismas que oscilaban entre 30\% y 49\% de los suicidas (Taborda y Téllez-Vargas, 2006); todas estas cifras son muy superiores a la media costarricense para la década, establecida en $12,05 \%$.

Por último, llama la atención la amplia gama de "problemas" a que se refieren los motivos del acto suicida (pasional, familiar, económico, personal, conyugal, de salud y con la justicia), puesto que McAuliffe, Corcoran, Keeley y Perry (2003), establecieron en una muestra de estudiantes universitarios de Irlanda, una asociación entre una pobre resolución de conflictos $y$ un aumento significativo del riesgo de presentar ideación suicida. 


\section{OCURRENCIA DEL FENÓMENO}

En cuanto al mes de ocurrencia del evento, la tabla 6 muestra un panorama bastante heterogéneo, sin perfilarse un patrón específico o particular en ese nivel.

TABLA 6

DISTRIBUCIÓN DE LA POBLACIÓN FALLECIDA POR SUICIDIO

POR MES, SEGÚN AÑO COSTA RICA, 2000-2009

(VALORES PORCENTUALES)

\begin{tabular}{lcccccccccc}
\hline \multicolumn{1}{c}{ MES } & 2000 & 2001 & 2002 & 2003 & 2004 & 2005 & 2006 & 2007 & 2008 & 2009 \\
\hline Enero & 7,2 & 7,3 & 9,9 & 8,2 & 9,0 & 6,5 & 9,0 & 8,2 & 11,2 & 10,2 \\
Febrero & 7,6 & 8,2 & 5,3 & 6,4 & 11,3 & 7,1 & 11,1 & 8,2 & 9,2 & 7,7 \\
Marzo & 9,1 & 11,3 & 7,1 & 8,5 & 8,7 & 10,5 & 8,4 & 7,3 & 6,6 & 8,6 \\
Abril & 11,1 & 8,2 & 8,5 & 9,1 & 6,8 & 9,3 & 6,8 & 6,0 & 7,6 & 7,7 \\
Mayo & 9,9 & 6,4 & 6,4 & 7,6 & 6,8 & 7,4 & 8,7 & 7,9 & 8,9 & 8,8 \\
Junio & 9,9 & 8,2 & 6,7 & 11,0 & 9,6 & 9,6 & 9,0 & 7,6 & 7,9 & 9,1 \\
Julio & 9,9 & 6,0 & 8,9 & 12,5 & 9,9 & 11,2 & 6,8 & 9,9 & 4,6 & 9,9 \\
Agosto & 6,1 & 11,8 & 8,2 & 7,9 & 8,4 & 8,4 & 6,3 & 9,6 & 9,5 & 6,4 \\
Setiembre & 7,6 & 7,3 & 8,5 & 8,5 & 6,5 & 7,4 & 10,0 & 9,9 & 7,9 & 9,7 \\
Octubre & 7,2 & 9,0 & 7,8 & 7,3 & 8,7 & 9,9 & 7,1 & 7,9 & 10,8 & 6,4 \\
Noviembre & 7,2 & 7,3 & 12,8 & 5,1 & 5,3 & 6,2 & 7,1 & 9,3 & 7,6 & 5,8 \\
Diciembre & 7,2 & 9,0 & 9,9 & 7,9 & 9,0 & 6,5 & 9,7 & 8,2 & 8,2 & 9,7 \\
\hline \multicolumn{1}{c}{ TOTAL } & $100 \%$ & $100 \%$ & $100 \%$ & $100 \%$ & $100 \%$ & $100 \%$ & $100 \%$ & $100 \%$ & $100 \%$ & $100 \%$ \\
\hline
\end{tabular}

Fuente: Anuarios de Estadísticas Policiales, Poder Judicial. En: <http://www.poder-judicial.go.cr/planificacion/Estadisticas/ policiales.html>

En concordancia con lo anterior, tampoco se muestra un perfil de ocurrencia por trimestre; en la figura 5 aparece la distribución trimestral de una manera relativamente heterogénea (ver figura 5).

El primer trimestre se presenta como el de mayor incidencia en cinco casos (2001, 2004, 2006, 2008 y 2009), seguido por el tercer trimestre en tres casos (2003, 2005 y 2007); mientras tanto, el segundo y el cuarto trimestre solo obtuvieron la mayor incidencia en uno de los casos (años 2000 y 2002 respectivamente).

$\mathrm{Al}$ agrupar los datos por semestre (figura 6) destaca, que si bien no se hallan diferencias abrumadoras, en siete de los diez años, más de la mitad de los suicidios se produjeron durante el primer semestre del año.

Respecto al día de la semana en que se produjo el suicidio (tabla 7), tampoco se encontró un patrón específico, aunque a excepción del año 2005, en los restantes se registró más de un $35 \%$ para los fines de semana (viernes, sábado y domingo).

Este elemento podría estar asociado con el hecho de que, generalmente, en estos periodos las personas tienen más tiempo para permanecer en sus hogares y estar consigo mismas $y$ mantienen un mayor consumo de bebidas alcohólicas, aspecto que tendría congruencia con el hecho de que este motivo registre un ascenso relativo en el periodo y con lo establecido en la literatura especializada (Bejarano y Sáenz, 2000; Matos et ál., 2001).

\section{EL GÉNERO COMO ELEMENTO INTERVINIENTE}

A pesar de observarse una tendencia al descenso en el caso de los varones y viceversa en cuanto a las mujeres (ver figura 7 ), en los últimos años de la década, dicha tendencia parece revertirse. 
FIGURA 5

DISTRIBUCIÓN DE LA POBLACIÓN FALLECIDA POR SUICIDIO, POR TRIMESTRE, SEGÚN AÑO

COSTA RICA, 2000-2009

(VALORES PORCENTUALES)

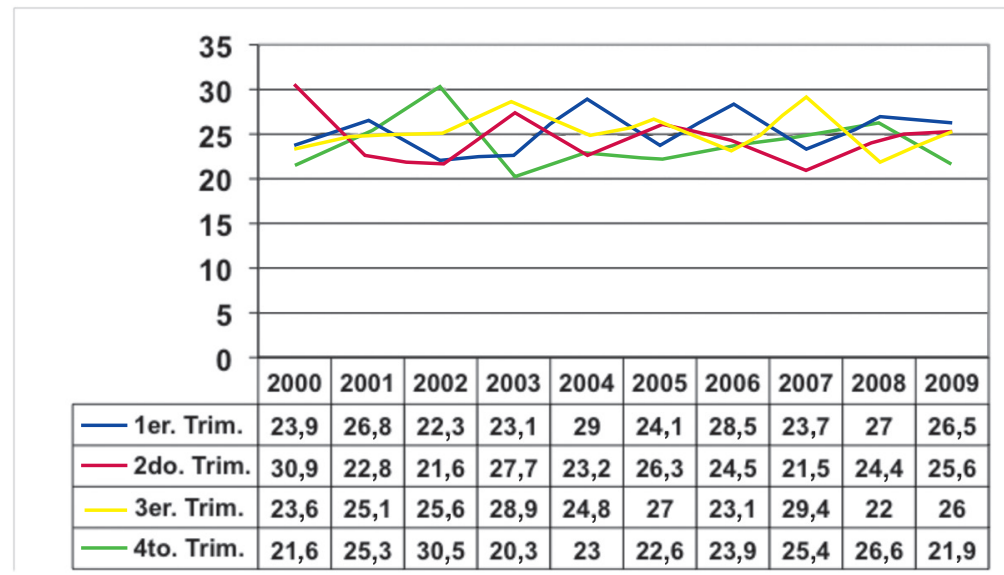

Fuente: Anuarios de Estadísticas Policiales, Poder Judicial. En: <http://www.poder-judicial.go.cr/planificacion/Estadisticas/ policiales.html>

FIGURA 6

DISTRIBUCIÓN DE LA POBLACIÓN FALLECIDA POR SUICIDIO, POR SEMESTRE, SEGÚN AÑO

COSTA RICA, 2000-2009

(VALORES PORCENTUALES)

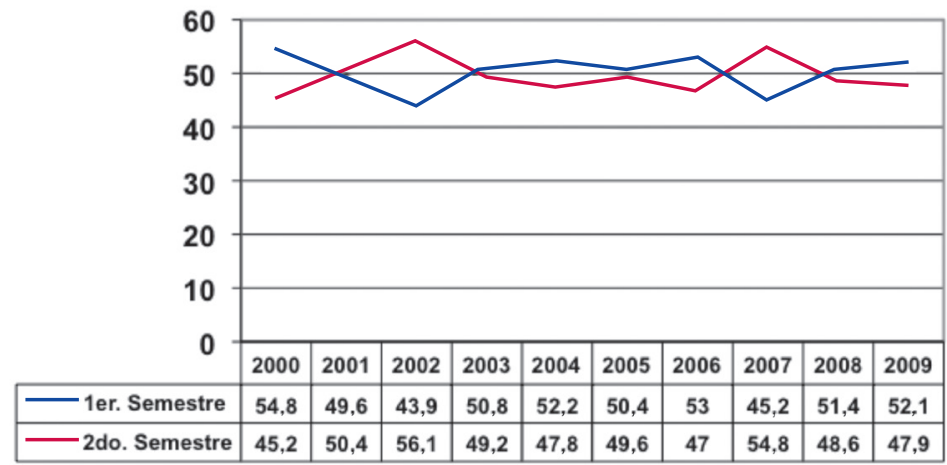

Fuente: Anuarios de Estadísticas Policiales, Poder Judicial. En: <http://www.poder-judicial.go.cr/planificacion/Estadisticas/ policiales.html> 
TABLA 7

DISTRIBUCIÓN DE LA POBLACIÓN FALLECIDA POR SUICIDIO, POR DÍA DE LA SEMANA, SEGÚN AÑO, COSTA RICA, 2000-2009

(VALORES PORCENTUALES)

\begin{tabular}{lcccccccccc}
\hline DÍA & 2000 & 2001 & 2002 & 2003 & 2004 & 2005 & 2006 & 2007 & 2008 & 2009 \\
\hline Lunes & 13,3 & 16,8 & 17,4 & 18,0 & 18,0 & 20,1 & 11,8 & 18,4 & 18,8 & 16,3 \\
Martes & 19,0 & 19,0 & 14,2 & 14,0 & 14,0 & 16,4 & 12,1 & 14,3 & 13,5 & 9,7 \\
Miércoles & 15,2 & 13,4 & 13,1 & 11,2 & 10,0 & 18,6 & 14,6 & 14,3 & 12,8 & 16,9 \\
Jueves & 12,9 & 13,4 & 10,6 & 14,9 & 13,0 & 18,0 & 17,6 & 11,1 & 11,2 & 12,4 \\
Viernes & 14,1 & 11,6 & 12,4 & 11,5 & 11,8 & 11,4 & 14,7 & 12,4 & 11,5 & 12,4 \\
Sábado & 12,9 & 15,9 & 16,7 & 15,2 & 17,4 & 12,7 & 11,1 & 13,6 & 15,8 & 15,7 \\
Domingo & 12,6 & 9,9 & 15,6 & 15,2 & 15,8 & 2,8 & 18,1 & 15,9 & 16,4 & 16,6 \\
\hline TOTAL & $100 \%$ & $100 \%$ & $100 \%$ & $100 \%$ & $100 \%$ & $100 \%$ & $100 \%$ & $100 \%$ & $100 \%$ & $100 \%$ \\
\hline
\end{tabular}

Fuente: Anuarios de Estadísticas Policiales, Poder Judicial. En: <http://www.poder-judicial.go.cr/planificacion/Estadisticas/ policiales.html>

FIGURA 7

DISTRIBUCIÓN DE LA POBLACIÓN FALLECIDA POR SUICIDIO, POR SEXO, SEGÚN AÑO

COSTA RICA, 2000-2009

(VALORES PORCENTUALES)

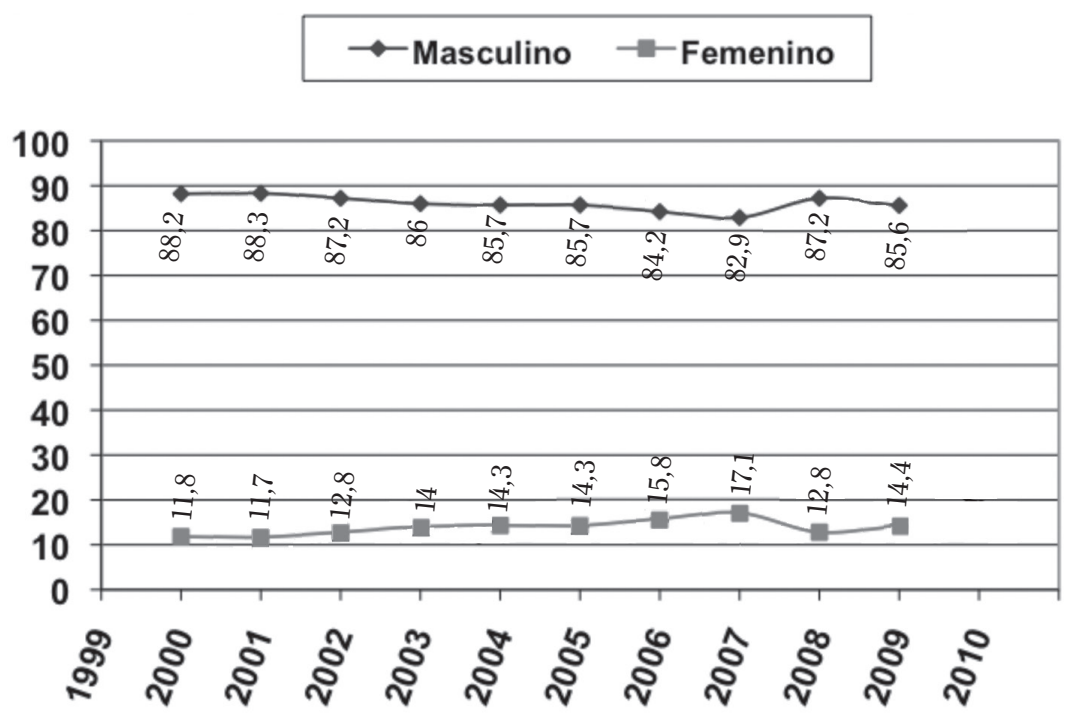

Fuente: Anuarios de Estadísticas Policiales. Poder Judicial. En: <http://www.poderjudicial.go.cr/planificacion/Estadisticas/policiales.html> 
El predominio tan marcado de los varones, no solo es coincidente con la información internacional y lo ocurrido en Costa Rica entre 1980 y 1994 (Granados, 1997), en tanto la consumación de los suicidios es considerablemente mayor en hombres; así, por ejemplo, en España se demostró una razón de 3:1 entre hombres y mujeres (74,64\% y 25,35\% respectivamente) para el periodo 1986-2002 (Arán et ál., 2006), mientras que en Colombia fue de 4:1 (González, 2006, citado en Taborda y Téllez-Vargas, 2006).

En la figura 8, se muestran las tasas por cien mil habitantes para cada sexo, aspecto en el que también se aprecia una marcada diferencia, estableciéndose una tasa anual promedio de 12,5 y 2,1 por cada cien mil habitantes varones y mujeres respectivamente, para una razón promedio durante la década de 5,95:1. Nótese que la tasa anual promedio para la población general en este periodo fue de 7,4 por cada cien mil habitantes, lo que indica que la tasa para los varones está cinco puntos por encima del global nacional.

Esta situación refleja el efecto de la socialización en torno a la construcción de la identidad masculina y su relación con la violencia, aspecto que también se refleja

FIGURA 8

TASA DE SUICIDIOS POR 100000 HABITANTES POR SEXO, SEGÚN AÑO

COSTA RICA, 2000-2009

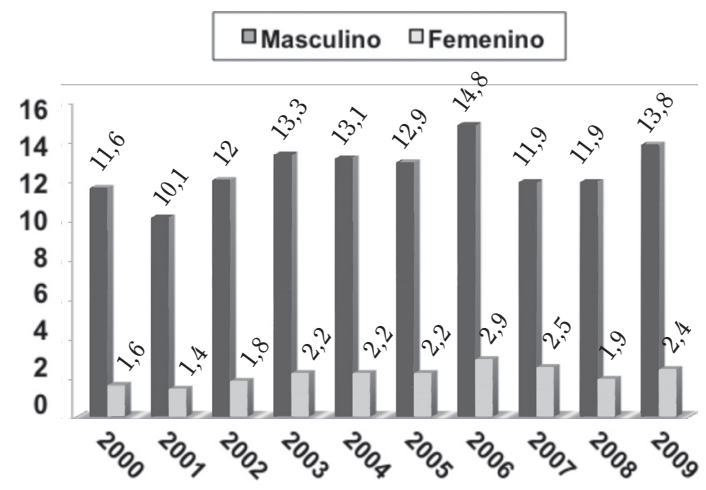

Fuente: Anuarios de Estadísticas Policiales. Poder Judicial. En: <http://www.poderjudicial.go.cr/planificacion/ Estadisticas/policiales.html> en porcentajes similares de hombres como autores y víctimas de homicidios dolosos - por ejemplo, 97,3\% y $82 \%$, respectivamente- (Sáenz, 2006). La asociación entre masculinidad $y$ violencia a lo largo del proceso de socialización humana, implica no solo que los varones lleguen a ser más explosivos, desplazando la agresión hacia el mundo externo, tanto en el plano intra como extra-familiar, sino que además, genera que lo dirijan contra sí-mismos, por ejemplo, el varón que comete femicidio y luego se suicida. En este sentido, llama la atención que, tal como plantean Tuesca y Navarro (2003), la literatura especializada señale la predominancia de las armas de fuego y el ahorcamiento como métodos utilizados por los hombres que cometen suicidio, los cuales aseguran mayor letalidad, mientras que las mujeres utilizan métodos más pasivos y menos violentos.

Aunado a ello, Arán et ál. (2006) plantean la vulnerabilidad ante determinados factores de riesgo a la cual están expuestos/as los y las adolescentes y jóvenes en función del sexo, tales como la depresión, los trastornos de conducta alimentaria o la forma de afrontar los problemas vitales.

\section{LOCALIZACIÓN GEOGRÁFICA COMO FACTOR DE RIESGO}

En la tabla 8 se presentan los datos registrados de la población fallecida por provincia $y$ año.

Congruente con el conglomerado poblacional del país, tal como se aprecia en la tabla 8, San José y Alajuela registran alrededor de la mitad de suicidios anuales, pues precisamente son las dos provincias de mayor población nacional, 35,47\% y 18,75\% respectivamente en relación con el censo de población de 2000 (Instituto Nacional de Estadística y Censos, 2001). No obstante, llama la atención que en varios años de la década analizada, las provincias de menor población, sean Puntarenas, Limón y Guanacaste, con un $9,36 \%, 8,91 \%$ y $6,92 \%$ de la población nacional en el censo de 2000, según corresponde, presenten mayores proporciones de suicidios que Cartago y Heredia, que son la tercera $y$ 
TABLA 8

DISTRIBUCIÓN DE LA POBLACIÓN FALLECIDA POR SUICIDIO, POR PROVINCIA, SEGÚN AÑO

COSTA RICA, 2000-2009

(VALORES PORCENTUALES)

\begin{tabular}{lcccccccccc}
\hline PROVINCIA & 2000 & 2001 & 2002 & 2003 & 2004 & 2005 & 2006 & 2007 & 2008 & 2009 \\
\hline San José & 38,4 & 33,6 & 36,9 & 32,8 & 39,1 & 30,0 & 30,8 & 30,2 & 34,8 & 31,8 \\
Alajuela & 17,5 & 14,7 & 14,2 & 18,9 & 16,4 & 17,3 & 20,8 & 17,8 & 12,2 & 18,8 \\
Cartago & 9,9 & 9,9 & 11,7 & 9,4 & 10,0 & 15,2 & 9,2 & 11,4 & 9,5 & 7,7 \\
Heredia & 9,1 & 6,9 & 7,1 & 7,0 & 9,0 & 6,8 & 9,0 & 7,9 & 7,6 & 6,9 \\
Guanacaste & 6,1 & 10,8 & 10,3 & 9,4 & 8,4 & 8,4 & 9,2 & 7,9 & 11,2 & 11,6 \\
Puntarenas & 6,1 & 12,5 & 9,9 & 14,0 & 10,0 & 9,9 & 9,2 & 12,7 & 12,2 & 13,0 \\
Limón & 12,9 & 11,6 & 9,9 & 8,5 & 7,1 & 12,4 & 11,8 & 12,1 & 12,5 & 10,2 \\
\hline TOTAL & $100 \%$ & $100 \%$ & $100 \%$ & $100 \%$ & $100 \%$ & $100 \%$ & $100 \%$ & $100 \%$ & $100 \%$ & $100 \%$ \\
\hline
\end{tabular}

Fuente: Anuarios de Estadísticas Policiales. Poder Judicial. En: <http://www.poder-judicial.go.cr/planificacion/ Estadisticas/policiales.html>

cuarta provincia en contenido poblacional con un $11,32 \%$ y $9,28 \%$ (Instituto Nacional de Estadística y Censos).

El hecho de que Guanacaste aparezca como la provincia con la mayor tasa promedio anual de suicidio a lo largo de la década (tabla 9 y figura 9), representa un cambio respecto al periodo 1980-1994, en que Limón ocupaba este lugar (Granados, 1998a), al igual que Puntarenas se ubique solo a 0,7 de ese segundo lugar. Lo anterior podría obedecer al radical cambio que ha presentado esta provincia (Guanacaste) en cuanto al impacto del turismo en su economía y su cultura. Aunado a ello, y a pesar de que Limón haya abandonado ese primer lugar, continúa ocupando un sitio destacado y por encima de la media nacional, aspecto de suma importancia si se analiza en relación con la tasa de homicidios dolosos por cada cien mil habitantes, donde ocupa el indiscutible primer lugar (Sáenz, 2006), lo cual apunta a la violencia contra otros o contra sí-mismo como elemento predominante de la interacción social en esta provincia.

En la tabla 9 resulta evidente que la segunda mitad de la década marca un significativo incremento en la tasa de suicidios en Guanacaste, Limón y Puntarenas, el cual influye en la tasa promedio anual de la década por provincia (figura 9).

TABLA 9

TASA DE SUICIDIOS POR 100000 HABITANTES, POR PROVINCIA, SEGÚN AÑO COSTA RICA, 2000-2009

\begin{tabular}{lcccccccccc}
\hline PROVINCIA & 2000 & 2001 & 2002 & 2003 & 2004 & 2005 & 2006 & 2007 & 2008 & 2009 \\
\hline San José & 7,3 & 5,5 & 7,3 & 7,6 & 8,6 & 6,6 & 7,8 & 6,3 & 6,9 & 7,4 \\
Alajuela & 6,2 & 4,5 & 5,3 & 8,0 & 6,8 & 7,0 & 9,8 & 6,9 & 4,5 & 8,1 \\
Cartago & 5,8 & 5,0 & 7,2 & 6,7 & 6,8 & 11,0 & 7,3 & 7,4 & 5,9 & 5,6 \\
Heredia & 6,6 & 4,3 & 5,3 & 6,0 & 7,5 & 6,0 & 8,6 & 6,2 & 5,7 & 6,1 \\
Guanacaste & 5,9 & 9,0 & 10,3 & 10,9 & 9,4 & 9,0 & 11,8 & 8,3 & 11,1 & 13,5 \\
Puntarenas & 4,3 & 7,7 & 7,3 & 11,9 & 8,1 & 8,0 & 8,6 & 9,7 & 8,8 & 11,1 \\
Limón & 9,7 & 7,5 & 7,7 & 7,6 & 6,1 & 11,0 & 11,6 & 9,6 & 9,4 & 9,1 \\
\hline Costa Rica & 6,7 & 5,8 & 6,9 & 7,9 & 7,7 & 7,7 & 8,9 & 7,3 & 6,9 & 8,2 \\
\hline
\end{tabular}

Fuente: Elaboración personal y Anuarios de Estadísticas Policiales, Poder Judicial. En: <http://www.poder-judicial.go.cr/planificacion/ Estadisticas/policiales.html> 
FIGURA 9

TASA PROMEDIO ANUAL DE SUICIDIOS POR 100000 HABITANTES

SEGÚN PROVINCIA

COSTA RICA, 2000-2009

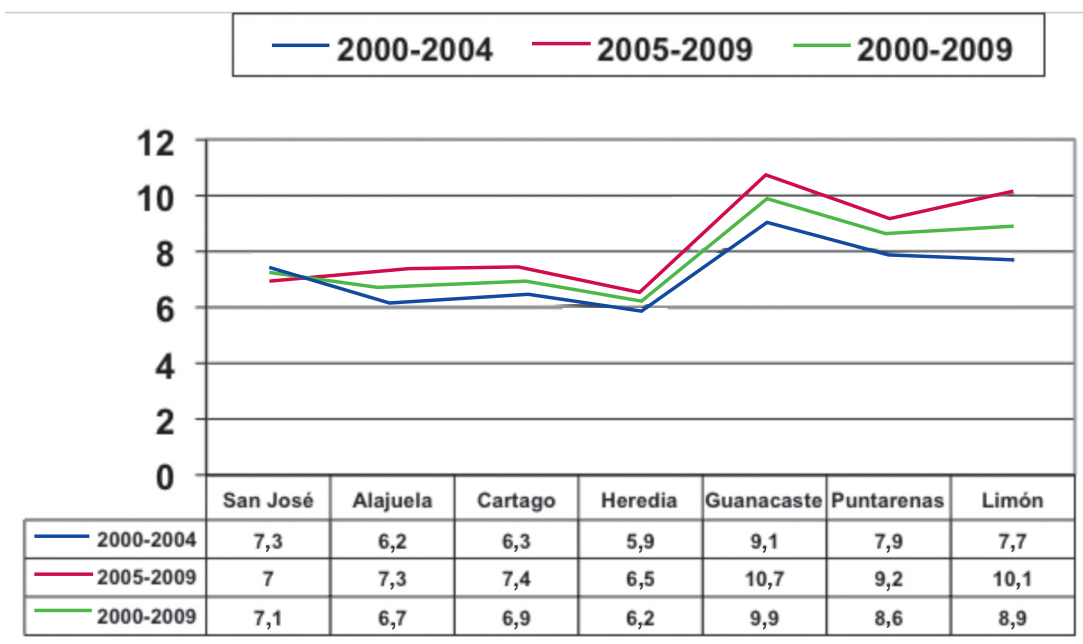

Fuente: Elaboración propia.

Denotándose que salvo el caso de la provincia de San José, dicho promedio anual de la década es inferior al promedio anual del segundo quinquenio en el resto de provincias, siendo imposible dejar de analizar este fenómeno desligado de la proporción de hogares pobres en que se han visto sumidas estas franjas de población. Mientras que para el año 2009 el país registró 18,5\% de hogares pobres para el territorio nacional, las regiones de planificación que abarcan estas provincias registraron porcentajes muy por encima: Huetar Atlántica (Limón) 21,6\%, Chorotega (Guanacaste) 24,1\% y, Pacífico Central y Brunca (Puntarenas) 28,8\%.

Aunado a ello, en 2009 estas cuatro regiones de planificación presentaron una tasa de desempleo abierto mayor al promedio nacional $(7,8)$, en su orden: Chorotega $(10,1)$, Pacífico Central $(8,2)$, Brunca $(8,2)$ y Huetar Atlántica $(7,9)$, lo que alude a una problemática social significativa de atender.

Particularmente, la situación de esas tres provincias se asocia con el hecho de que, a su vez, para el año 2009 registraban una tasa bruta de mortalidad superiores al promedio nacional, a saber: Puntarenas (5,03), Limón $(4,65)$ y Guanacaste $(3,94)$.

\section{DISCUSIÓN}

A lo largo de la década fallecieron por suicidio 3112 personas, lo cual amerita formularse una pregunta: ¿por qué se suicidan las personas? Sin particularizar en grupos específicos esta pregunta genérica es una interrogante fundamental para direccionar la prevención específica y la promoción de la salud mental, así como, para la generación de una legislación adecuada y una política pública en esta materia. Llama la atención que a pesar de existir una Ley General de Salud (Ley 5395, del 30 de octubre de 1973 y sus reformas), esta se concentre en aspectos fundamentalmente biologicistas $y$ deje de lado la salud mental y emocional de los habitantes de la República.

De esta manera, se hace urgente responder a la necesidad de una política de salud mental en el nivel nacional, que integre programas preventivos en las regiones y grupos etarios de más alto riesgo, particularizando en 
las provincias que registran mayores niveles de pobreza. En este sentido:

... la prevención del suicidio y la atención de las personas que intentan suicidarse, al igual que la atención de las familias afectadas por las repercusiones, forman en muchos países parte importante de los programas de salud mental. La importancia de los factores psicológicos y psicosociales que influyen en su comisión y los efectos sobre los sobrevivientes justifican ampliamente su inclusión (Macanche, González y Cruz, 2004).

En este sentido, llama la atención el reportaje del periodista Carlos Arguedas, publicado en el diario La Nación del 6 de febrero de 2011, página $12 \mathrm{~A}$, en el cual se evidencia que el país no cuenta con programas de ayuda para jóvenes que presentan riesgo de suicidio, por lo cual, a la Caja Costarricense de Seguro Social le urge la contratación de un mayor número de profesionales en el ámbito de la salud mental con el propósito de brindar una atención inmediata de las referencias por problemas emocionales $y$ no brindar citas a seis meses o un año plazo como sucede en la actualidad.

Paralelamente, en cuanto a los motivos aparentes del evento suicida, sería importante profundizar los datos numéricos mediante la utilización de métodos cualitativos, como la autopsia psicológica, también se podría recurrir a herramientas como la entrevista en profundidad con familiares, compañeros y amigos de la víctima, así como, el análisis documental de expedientes (médico, laboral, educativo, judicial); esto implicaría una directriz para que la Sección de Psiquiatría y Psicología Forense del Poder Judicial implantara este procedimiento en todos los casos de posible suicidio, amparada en las recomendaciones $y$ acciones puntuales, que desde el punto de vista preventivo se podrían tomar.

Otro tema trascendental es la relación entre la construcción de la masculinidad y la violencia, puesto que para la década en estudio, al menos por cada mujer que se suicidó lo hicieron seis varones y urge al sector educación efectuar transformaciones radicales en materia de equidad de género, pero también en cuanto al papel de las y los docentes como generadores de salud mental. Esto implica un sistema educativo preocupado por la formación de niños, niñas $y$ adolescentes, procurando romper ese vínculo y no por el cumplimiento de programas escolares, que cada vez presentan más exigencias intelectuales y más dificultades, contribuyendo en mayor medida a la "robotización" de las y los estudiantes.

Aunado a ello, urge al sistema educativo centrarse en el entrenamiento de habilidades para la vida, las cuales pueden entenderse como competencias psicosociales que le permiten a los seres humanos enfrentar de manera positiva $y$ fructífera las demandas, desafíos y condiciones adversas del diario vivir, favoreciendo así una adaptación activa a su entorno social (Sáenz, 2001), no solo para generar estilos de vida saludables, sino también una estrategia de promoción de la salud mental, pues de acuerdo con este autor el modelo referido abarcaría las siguientes habilidades: conocimiento de sí-mismo, empatía, comunicación asertiva, relaciones interpersonales, toma de decisiones, resolución de conflictos, pensamiento creativo, pensamiento crítico, manejo de sentimientos y emociones, así como, manejo de estrés y tensiones.

Asimismo, resulta imperioso el desarrollo de una política de investigación en esta materia para develar desde una perspectiva cualitativa, la dinámica de este fenómeno en las diferentes zonas, grupos etarios e inclusive, de acuerdo con el sexo $y$ otras variables sociodemográficas. Consecuentemente, dicha política tendría como finalidad brindarle sentido a los números (Sáenz, 2007) que las tradicionales investigaciones epidemiológicas brindan. Así, surgen varias inquietudes: ¿por qué los suicidios llevan una tasa ascendente en Costa Rica?, ¿qué hay de particular en Guanacaste, Limón y Puntarenas para que registren las mayores tasas de suicidio por provincia?, ¿cómo influyen los indicadores sociales de las regiones y zonas del país en 
la génesis del suicidio?, ¿cómo se articula la construcción de la masculinidad con el hecho de dirigir la violencia contra sí-mismo?, ¿qué sucede con las personas que se ubican en periodos de edad teóricamente asociados con mayor estabilidad emocional y la consolidación de un proyecto de vida como para que mayoritariamente se suiciden?, ¿cómo se relaciona la construcción de la subjetividad con la génesis del suicidio?, entre otros cuestionamientos.

\section{REFERENCIAS}

Arán, M.; Gispert, R.; Puig, X.; Freitas, A. et ál. "Evolución temporal y distribución geográfica de la mortalidad por suicidio en Cataluña y España (1986-2002)". Gaceta Sanitaria 20 (6). 2006: 473-480.

Bejarano, J. y Sáenz, M. A. "Consumo de drogas y muerte violenta en Costa Rica". Adicciones 12 (3). 2000: 435-441.

Bonilla, M. y Sáenz, M. A. "Representaciones sociales en Costa Rica sobre personas nicaragüenses: un acercamiento desde los chistes populares". Humanitas 7 . 2010: 72-102.

Chanto, J. D. "Creencias racionales e irracionales sobre la vida y la muerte $y$ conductas suicidas: el caso de los adolescentes". Revista Costarricense de Psicología 36-37. 2004-2005: 23-33.

Díaz, O. y Sanabria, M. A. "El fenómeno de la muerte en el campo de la toxicomanía". Revista Española de Drogodependencias 19 (3). 1994: 235-244.

Goldston, D. B.; Reboussin, B. A. y Sergent, S. "Predictors of suicide attempts: state and trait components". Journal of Abnormal Psychology 115 (4). 2006: 842-849.

Granados, D. "Magnitud, estructura por edad $y$ sexo y tendencia del suicidio en Costa Rica, 1980-1994". Revista Costarricense de Salud Pública 11. 1997: 1-9.

Granados, D. "Magnitud y tendencia del suicidio en las provincias de Costa Rica, 1980-1994". Revista Costarricense de Salud Pública 12. 1998a: 1-22.

Granados, D. "Magnitud y tendencia del suicidio en los cantones de Costa Rica,
1980-1994". Revista Costarricense de Salud Pública 12. 1998b: 23-35.

Gutiérrez-García, A. G.; Contreras, C. M. y Orozco-Rodríguez, R. Ch. "El suicidio, conceptos actuales". Salud Mental 29 (5). 2006: 66-74.

Instituto Nacional de Estadística y Censos. IX Censo Nacional de Población y $\mathrm{V}$ de Vivienda. Costa Rica: cifras provisionales por provincia, cantón y distrito. San José: INEC, 2001.

Krug, E. G. et ál. (eds.) Informe mundial sobre la violencia y la salud. Washington DC: Organización Panamericana de la Salud, 2003

Lastra, I. et ál. "Prevención de las conductas suicidas”. J. L. Vázquez-Barquero (ed.). Psiquiatría en Atención Primaria. 2a reimpresión. Madrid, España. Grupo Aula Abierta, 1999: 753-770.

Lester, D. "Communitarianism and suicide prevention: proposals for the year 2000". Crisis: The Journal of the Crisis Intervention and Suicide Prevention 18 (3). 1997: 118-123.

Macanche, C.; González, R. y Cruz, M. Estado actual de la salud mental en Costa Rica. San José: Ministerio de SaludOrganización Panamericana de la Salud, 2004.

Matos, R.; Betancourt, A. et ál. "Muertes violentas y consumo de alcohol". Adicciones 13 (1). 2001: 75-80.

McAuliffe, C.; Corcoran, P.; Keeley, H. S. y Perry, I. J. "Risk of suicide ideation associated with problem-solving ability and attitudes toward suicidal behavior in university students". Crisis: The Journal of the Crisis Intervention and Suicide Prevention 24 (4). 2003: 160-167.

Méndez, F. y Araya, O. M. Evolución de la esperanza de vida al nacimiento en Costa Rica 1900-2000. San José: INEC, 2001.

Moyano, E. y Barria, R. "Suicidio y Producto Interno Bruto (PIB) en Chile: hacia un modelo predictivo". Revista Latinoamericana de Psicología 38 (2). 2006: 343-359. 
Proyecto Estado de la Nación. Estado de la Nación en Desarrollo Humano Sostenible: un análisis amplio y objetivo sobre la Costa Rica que tenemos, a partir de los indicadores más actuales (2009). San José: Editorama, 2010.

Sáenz, M. A. “'La Aventura de la Vida': un programa de prevención de drogas en América Latina”. Revista Costarricense de Psicología 18-19. 2001: 40-51.

Sáenz, M. A. "Los homicidios dolosos en Costa Rica: caracterización sociodemográfica". Revista de Ciencias Sociales 111-112. Universidad de Costa Rica, 2006: 177-188.

Sáenz, M. A. "Aproximación a la investigación cualitativa en Psicología y Educación". Humanitas 4. 2007: 63-86.

Taborda, L. C. y Téllez-Vargas, J. "El suicidio en cifras”. En: J. Téllez-Vargas y J. Forero (eds.). Suicidio: Neurobiología, factores de riesgo y prevención. (s. f.). [En línea]
En: <http://www.psiquiatriabiologica. org.co/publicaciones/suicidio.html> (Recuperado el 17 de enero de 2010).

Tamarit, J. M. "Introducción". J. M. Tamarit (coord.). Victimas olvidadas. Valencia, España: Tirant lo Blanch. 2010: 9-22.

Torres de Galvis, Y. "Método epidemiológico aplicado a la Farmacodependencia". Jornadas de actualización sobre uso $y$ abuso de alcohol y drogas. Caracas, Venezuela. 1990.

Tuesca, R. y Navarro, E. "Factores de riesgo asociados al suicido e intento de suicidio". Salud Uninorte 17. 2003: 19-28.

Vargas, L. "Suicidio en niños y adolescentes". Revista Costarricense de Psicología 28. 1997: 63-74.

Fecha de ingreso: 10/02/2011

Fecha de aprobación: 19/05/2011 
